\title{
Design and Performance Estimation of a Photonic Integrated Beamforming Receiver for Scan-On- Receive Synthetic Aperture Radar
}

\author{
M. Reza, G. Serafino, T. Otto, A. Mohammad, H. Mohammadhosseini, L. Shiramin, F. Floris, M. \\ Kolb, D. Bail, S. Gabrielli, C. Roeloffzen, P. van Dijk, A. Abbasi, B. Desoete, P. Ghelfi
}

\begin{abstract}
Synthetic aperture radar is a remote sensing technology finding applications in a wide range of fields, especially related to Earth observation. It enables a fine imaging that is crucial in critical activities, like environmental monitoring for natural resource management or disasters prevention. In this picture, the scan-on-receive paradigm allows for enhanced imaging capabilities thanks to wide swath observations at finer azimuthal resolution achieved by beamforming of multiple simultaneous antenna beams. Recently, solutions based on microwave photonics techniques demonstrated the possibility of an efficient implementation of beamforming, overcoming some limitations posed by purely electronic solutions, offering unprecedented flexibility and precision to RF systems. Moreover, photonics-assisted RF beamformers can nowadays be realized as integrated circuits, with reduced size and power consumption with respect to digital beamforming approaches. This paper presents the design analysis and the challenges of the development of a hybrid photonicintegrated circuit as the core element of an X-band scanon-receive spaceborne synthetic aperture radar. The proposed photonic-integrated circuit synthetizes three simultaneous scanning beams on the received signal, and performs the frequency down-conversion, guaranteeing a compact $15 \mathrm{~cm}^{2}$-form factor, less than $6 \mathrm{~W}$ power consumption, and $55 \mathrm{~dB}$ of dynamic range. The whole photonics-assisted system is designed for space compliance and meets the target application requirements, representing a step forward toward a deeper penetration of photonics in microwave applications for challenging scenarios, like the observation of the Earth from space.
\end{abstract}

Index Terms - Beamforming, photonic integrated circuit, hybrid integration, synthetic aperture radar.

\section{INTRODUCTION}

Z ARTH Observation (EO) from Space has been a key - tool for decades in traditional applications like cartography and elevation mapping [1]. However, the impact of climate changes on human life and activities has recently become more important, revealing the crucial importance of EO also to understand the effects of global warming and for their mitigation, thanks to environmental monitoring for disaster prevention [2]. More in general, it allows for risk management, (volcanic activity, earthquakes, landslides, droughts, floods), situation awareness and access control in case of natural disasters, pollution monitoring and surveillance (oil spill, leak detection), resource management (biomass, oil, gas), agriculture (vegetation type, coverage). In this scenario, EO systems continuously face the demand for increased performance, particularly in terms of resolution and sensitivity.

Spaceborne Synthetic Aperture Radars (SARs) are an established technology in EO, a class of radars that offers a fine imaging resolution by processing sets of consecutive acquisitions recorded while flying over the targeted area [3]. Although the SAR is a very mature technology, research keeps on seeking innovative techniques to improve its performance. In particular, a recent advanced technique is the scan-onreceive (SCORE) mode. Indeed, the SCORE-SAR paradigm allows overcoming a fundamental limitation of the conventional stripmap SAR, where the achievable spatial resolution along-track is inversely related to the swath width across-track and hence requesting multiple passes of the satellite to fully observe a given area with a high resolution. The SCORE principle consists in illuminating a wide swath in transmission, and receiving the radar echoes through multiple smaller (i.e., higher-gain) receive beams steered across-track to cover the whole swath. The implementation of SCORE is currently studied in detail for a large number of science
This work was supported by the EC through the H2020 project SPACEBEAM (grant no. 870421)

M. Reza and G. Serafino are with Scuola Superiore Sant'Anna, TeCIP Institute, 56124 Pisa, Italy (manuel.reza@santannapisa.it)

T. Otto and S. Gabrielli are with OHB System AG, D-28359 Bremen, Germany (tobias.otto@ohb.de)

A. Mohammad, C. Roeloffzen, and P, van Djik are with LioniX International, Enschede, The Netherlands (a.w.m.mohammad@lionixint.com)
H. Mohammadhosseini, L. Shiramin, D. Bale, A. Abbasi, and B. Desoete are with Antwerp Space NV, B-2660 Antwerp, Belgium (hakimeh.mohammadhosseini@antwerpspace.be)

F. Floris is with Tyndall National Institute - University College Cork, Cork, Ireland (francesco.floris@tyndall.ie)

M. Kolb is with Astro- und Feinwerktechnik Adlershof GmbH, 12489 Berlin, Germany (m.kolb@astrofein.com)

P. Ghelfi is with CNIT - PNTLab, 56124 Pisa, Italy, and with Scuola Superiore Sant'Anna, TeCIP Institute, 56124 Pisa, Italy (paolo.ghelfi@cnit.it) 


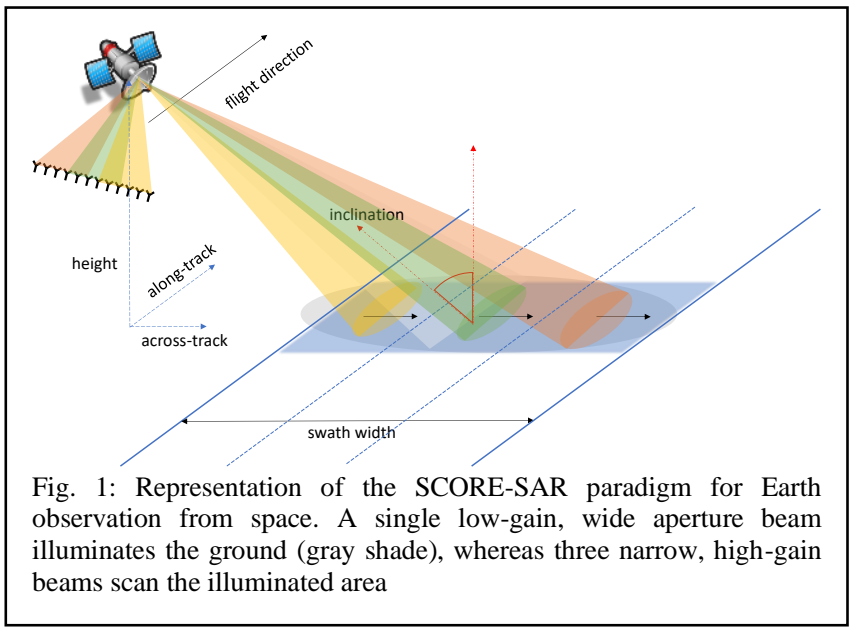

missions. Currently, no space-born mission with operational SCORE-SAR paradigm is present. To the Authors knowledge, the first mission which implements the SCORE-SAR using Field Programmable Gate Array (FPGA) technology will be the NISAR Mission planned to be launched at the end of 2022 [4]. To date, there are no X-band SCORE SAR systems in orbit or planned, which is mainly related to the limitations of digital electronics in enabling multi-channel, wide bandwidth, real-time digital beamforming (from few hundreds of $\mathrm{MHz}$ up to $1.2 \mathrm{GHz}$ per channel) in terms of size, weight, power, and costs (SWaP-C), preventing its applicability on board of small and micro-satellites [5], [6].

To make the SCORE-SAR observation mode also available for smaller SAR missions, a compact and less power-hungry alternative implementation of the on-board beamforming is required. Photonic technologies are providing a leap forward in the field of satellite applications. Due to their broadband nature, insensitivity to the electromagnetic interference (EMI), low loss, and data-transparent link capacity, they have already penetrated various satellite subsystems and functions [7]. Moreover, microwave photonics (i.e., photonics applied to microwave systems) offer the possibility of easily performing frequency-agnostic operations to process RF signals in any employed region of the RF spectrum. Microwave photonic technologies have already demonstrated their capability of supporting the development of microwave remote sensing systems with enhanced performance characterized by unprecedented flexibility [8], [9]. In addition, the impressive latest advances in photonic integrated technologies are very promising in reducing the SWaP budget of microwave systems [10], currently providing a technological maturity suitable for space applications.

TABLE I

BENCHMARK COMPARISON BETWEEN DIGITAL-BASED AND PHOTONIC-BASED SCORE-SAR RECEIVER

\begin{tabular}{|c|c|c|}
\hline Parameter & Digital-based & Photonics-based \\
\hline Frequency band & X-band $(9.3-9.9 \mathrm{GHz})$ & $5-40 \mathrm{GHz}$ \\
\hline Signal bandwidth & $\mathrm{Up}$ to $600 \mathrm{MHz}$ & Any RF bandwidth \\
\hline $\mathrm{N}^{\circ}$ input channels & 6 & $>12$ \\
\hline $\mathrm{N}^{\circ}$ output channels & 3 & 3 \\
\hline Footprint & $<20 \mathrm{dm}^{3}$ & $<2.5 \mathrm{dm}^{3}$ \\
\hline Mass & $<25 \mathrm{Kg}$ & $<4 \mathrm{Kg}$ \\
\hline Power consumption & $<120 \mathrm{~W}$ & $<6 \mathrm{~W}$ \\
\hline $\begin{array}{l}\text { Reconfiguration } \\
\text { dynamics }\end{array}$ & Discrete & Continuous \\
\hline
\end{tabular}

Photonic integrated circuit (PIC) technologies have demonstrated to be capable of implementing OBF networks (OBFNs) for 5G communications [11] and wideband radar systems [12]. Therefore, photonics can be considered as a key enabling technology for developing more flexible spaceborne SAR systems. Nevertheless, the development of on-board subsystems based on photonics integrated devices for space applications is still an open research field [13].

This paper describes the advances in the design and development of the first photonics-assisted SCORE-SAR receiver module intended for EO from space. This activity is carried out within the SPACEBEAM project (Space SAR system with reconfigurable integrated photonic beamforming) [14]. The core of the system is an extremely compact hybrid PIC, realized thanks to the advanced hybrid integration of a $\mathrm{Si}_{3} \mathrm{~N}_{4}$ TriPleX ${ }^{\mathrm{TM}}$ platform chip, used for the realization of extremely low-loss passive components, with InP chips for active and opto-electronic components. The proposed SCORE-SAR photonics-assisted receiver is able to process 12 incoming RF signals in the optical domain, thanks to the implementation of a reconfigurable optical analog Blass matrix [15], [16] capable of dynamically synthetize one, two, or three beams with continuity. Simultaneously, the PIC, by using an optical heterodyne detection scheme, is also able to perform the down-conversion to intermediate frequency (IF) of the X-band signals received by the feed array elements (FAEs) of the antenna. Thanks to the photonic integrated technologies, such a complex receiver module still presents very low power consumption and extremely compact form factor. While the system has been designed for the X-band, its approach can be easily extended to higher frequencies, e.g. Ka-band, thanks to the large electro-optical bandwidth of photonic devices. The novelty of this work lays in designing a photonic integrated system implementing the SCORE-SAR paradigm with realistic EO mission requirements, through an unprecedented multiple-input multiple-output analog optical beamforming matrix.

The paper is divided in two parts, each one further subdivided in different sections. The first part introduces the SCORE-SAR system and it derives all the requirements that the proposed photonic beamformer needs to meet, with a topdown approach starting from the application (i.e. Earth observation) to the receiver processing unit. The second part describes the technology, the design, and the analysis of the novel integrated photonic beamformer. Moreover, the performance analysis of the beamformer architecture from a microwave perspective is carried out to evaluate its impact on the entire receiver system.

\section{SCORE-SAR SYSTEM ARCHITECTURE AND REQUIREMENTS}

The requirements on the PIC of the SCORE-SAR receiver directly derive from the system requirements. Therefore, a brief description of the full system is laid out: the SCORESAR principle is described, introducing the terminology and the main parameters. Then, the evaluation of the concept for the space mission applications is presented, which defines the requirements for the whole system. 


\begin{tabular}{ll}
\hline \multicolumn{1}{c}{ TABLE II } \\
\multicolumn{1}{c}{ SAR PAYLOAD END-TO-END REQUIREMENTS } \\
\hline \hline \multicolumn{1}{c}{ Parameter } & \multicolumn{1}{c}{ Value / Range } \\
\hline Frequency Band & \multicolumn{1}{c}{ X-Band $(9.6 \quad \mathrm{GHz} \quad$ center } \\
& frequency) \\
Orbit Height & LEO $(400-800 \mathrm{~km})$ \\
Incidence Angle & $>20^{\circ}$ \\
Swath Width (across-track) & $>30 \mathrm{~km} /$ goal: $50 \mathrm{~km}$ \\
Swath Length (along-track) & $>30 \mathrm{~km} /$ goal: $50 \mathrm{~km}$ \\
Ground Resolution & $<1.5 \mathrm{~m} \times 1.5 \mathrm{~m}$ \\
Noise Equivalent Sigma Zero & $<-20 \mathrm{~dB}$ \\
NESZ (Sensitivity) & $>32.5 \mathrm{~dB}$ \\
Dynamic Range & $<-20 \mathrm{~dB}$ \\
Ambiguity to Signal Ratio (ASR) &
\end{tabular}

\section{A. SCORE-SAR Principle and Technology}

A Synthetic Aperture Radar is an imaging radar mounted on a moving platform, from where electromagnetic waves are sequentially transmitted and the backscattered echoes are collected by the radar antenna. Therefore, consecutive times of transmission/reception corresponds to different positions due to the platform movement. By exploiting the phase coherence between multiple pulses of the consecutive acquisitions during the satellite flight, a virtual (synthetic) along-track (i.e. azimuth) aperture much longer than the real antenna aperture is obtained. By increasing the number of acquisitions (i.e., increasing the pulse repetition frequency, PRF), finer azimuth resolutions than simple side-looking airborne radars can be achieved [17], whereas the range resolution (perpendicular to the satellite motion, or acrosstrack) remains the same, as it depends only on the bandwidth of the transmitted chirped waveform.

Conventional stripmap SAR systems image a single swath over a continuous strip aligned with the satellite travel direction. This swath width in the range direction is limited by the unambiguous echo window $c /$ PRF. Therefore, higher PRF also means shorter pulse repetition interval (PRI), and hence narrower swath width. To decouple the conflicting azimuth resolution and the swath width parameters from the PRF, the SCORE technique reckons on the illumination of a wide area with a single low-directivity beam in transmit mode, whereas multiple high-gain beams are simultaneously steered in the across-track direction to receive the backscattered signal [18], [19]. An illustration of the SCORESAR concept is shown in Fig. 1. SCORE overcomes the limitations related to large swath width by allowing the system to acquire echoes from ambiguous regions (subswath) of the imaged area and assigning each one of those narrower sub-swaths to a specific $\mathrm{Rx}$ beam. The principle is based on the synthesis of multiple high-gain Rx beams that scan the echoes on the ground range. However, the price to pay for decoupling the swath-width from the azimuth resolution is an increase of SAR instrument architecture complexity, due to the need to steer the multiple Rx beams.

In current state-of-the-art beamforming solutions for space applications, the commonly adopted approach to beamforming leverages on FPGA digital signal processing (DSP) [20]. Moreover, the antenna signals from the FAEs are down-converted using RF mixers to frequencies suitable for digitalization, before the FPGA signal processing
TABLE III

ORBIT, SAR ANTENNA AND RECEIVER LINK SPECIFICATIONS

\begin{tabular}{ll}
\hline \multicolumn{1}{c}{ Parameter } & \multicolumn{1}{c}{ Value / Range } \\
\hline Orbit Height & $500 \mathrm{~km}$ \\
Antenna Type & Focal Plane Array with Reflector \\
Number of elements & 12 \\
Elements spacing & $0.89 \lambda$ \\
Tx incidence angle & $22.67^{\circ}$ \\
Tx Gain / Rx Gain & $>43 \mathrm{~dB} />47 \mathrm{~dB}$ \\
Tx Elevation Beamwidth & $5^{\circ}$ \\
Rx Scan Range & $-2.5^{\circ}$ to $2.5^{\circ}$ \\
Tx/Rx Azimuth Beamwidth & $0.7^{\circ}$ \\
Rx Elevation Beamwidth & $0.93^{\circ}$ \\
RF Bandwidth & $390 \mathrm{MHz}$ \\
Receiver Link Dynamic Range & $>32.5 \mathrm{~dB}$ \\
Receiver Link Input RF Power & $-89.6 \mathrm{dBm}$ to $-57.1 \mathrm{dBm}$ \\
\hline
\end{tabular}

beamforming. However, these systems usually require heavy payloads that are exclusive of large satellites only. On the other hand, a more compact and miniaturized radar system can fully exploit the advantages of the PIC technology with reduced SWaP. PICs can process all signals in analog form, including continuous beamforming and extremely flexible frequency down-conversion, using on-chip microwave photonic building blocks, such as real-time tunable couplers, combiners, phase controls, and filters. The significant reduction in SWaP that integrated photonics can offer compared to classic digital solutions is expected to enable the implementation of SCORE-SAR systems suitable for spaceborne missions. To the Authors knowledge, there is no current mission to date equipped with a SCORE-SAR system in orbit. Therefore, in TABLE I, the comparison is made between current state-of-the-art digital beamformers and the here-proposed photonic beamformer described in this work are compared. Apparently, the microwave photonics solution offers better performance in terms of flexibility and SWaP reduction. The SPACEBEAM photonic beamformer aims to achieve comparable or improved performance with respect to standard digital beamformers, demonstrating its suitability for space applications.

\section{B. System requirements}

Based on system specifications from previous space missions, the goal of the SPACEBEAM project is to develop a SCORE-SAR system with a swath 5 times wider than current spaceborne SAR systems, enabling a larger coverage up a $50-\mathrm{km}$ swath width, at the same time guaranteeing a $1.5-\mathrm{m}$ spatial resolution on both along- and across-track directions. The sensitivity and dynamic range of the system were considered to be such that it can be used to observe natural and man-made targets on a wide variety of applications such as agriculture, land-use monitoring, flood mapping and digital elevation model generation, to name few examples.

The expected dynamic range can be estimated by the scattering characteristics of the different kind of terrains: soils and rocks present the highest backscatter coefficient with a value of $10 \mathrm{~dB}$, whereas roads have the lowest one with -20 $\mathrm{dB}$ [21]. Including also $2.5 \mathrm{~dB}$ from free propagation loss in the atmosphere, the system needs to handle a dynamic range higher than $32.5 \mathrm{~dB}$. 


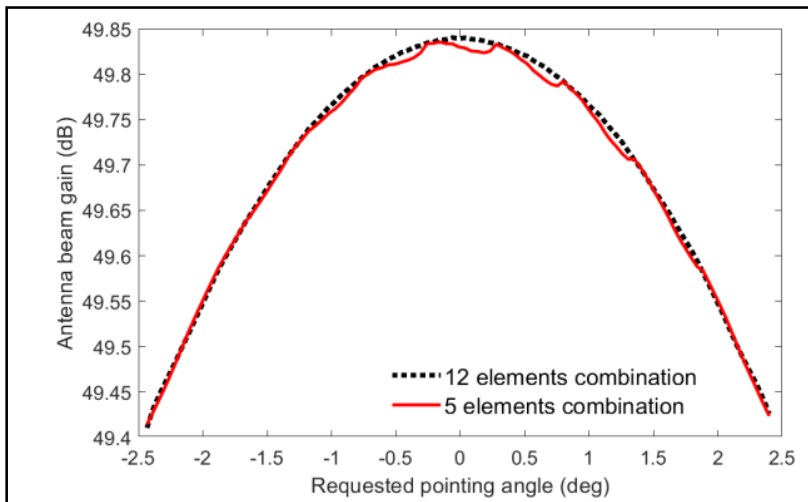

Fig. 2: Comparison of the antenna gain profile between whole feed combination and cluster-based solution.

The lowest backscatter coefficient, combined with the requirement on resolution, also sets the requirement for the sensitivity, quantified by the Noise Equivalent Sigma Zero (NESZ), which, for the current application, needs to be less than $-20 \mathrm{~dB}$. All the main system requirements are summarized in TABLE II. Once the main application requirements have been defined, it has been possible to design the antenna and receiver system.

\section{Antenna and receiver specifications}

The reference satellite is considered in the low Earth orbit (LEO) and will mount a $2.9 \mathrm{~m}$ diameter parabolic reflector antenna with a feed horn for the transmit beam and a focal plane array (FPA) feed composed by 12 FAEs elements spaced of $0.89 \lambda$ for the receiving beam, where $\lambda$ is the radar signal wavelength. The number of elements and their spacing have been selected as a trade-off between the required beamwidth (which determines the swath width) and the acceptable levels of sidelobes that can be rejected by the spatial filtering introduced by the parabolic reflector. In transmission, this antenna configuration has a beamwidth of $5^{\circ}$ and it is capable of covering the full $50 \mathrm{~km}$ swath width from a $500 \mathrm{~km}$ orbit height with an incidence angle of $22.67^{\circ}$. In reception, the antenna guarantees an along-track resolution $<1.15 \mathrm{~m}$. For this reason, to have a matching across-track resolution, an RF signal with $390 \mathrm{MHz}$ bandwidth is transmitted. It is worth noticing that the OBFN does not significantly affect the overall radiation pattern shape, since this mainly depends on the reflector and its geometry.

As for the frequency band, although the PIC beamformer can cover a huge range of frequencies, a SAR in the X-band (namely, at $9.6 \mathrm{GHz}$ ) is considered for the implementation of the project demonstrator. The RF front-end of the beamformer provides a local oscillator (LO) at $8.3 \mathrm{GHz}$, expecting a down-converted signal at an intermediate frequency (IF) of $1.3 \mathrm{GHz}$. Considering the observation geometry and the expected $32.5 \mathrm{~dB}$ dynamic range, the estimated received power at each FAE is expected to be in the range from $-90 \mathrm{dBm}$ to $-58 \mathrm{dBm}$. The synthetized $\mathrm{Rx}$ multiple beams should scan over the same Tx range of $5^{\circ}$ (from $-2.5^{\circ}$ to $2.5^{\circ}$ ) between two transmit events (i.e., within one PRI). The specifications of the antenna and the receiver are summarized in TABLE III, whereas the requirements on the beam formation are derived in the next section.
TABLE IV

SCAN-ON-RECEIVE BEAMFORMING REQUIREMENTS

\begin{tabular}{ll}
\hline \multicolumn{1}{c}{ Parameter } & Value / Range \\
\hline Nr. Rx Beams & 1 to 3 \\
Nr. Feed Array Elements per Beam & 5 of 12 \\
Nr. Beam directions & 50 \\
Dwell time (observation time) & $2.6 \mu \mathrm{s}$ \\
Beamforming weights amplitude rel. error & $<5 \%$ \\
Beamforming weights phase error & $<10^{\circ}$ \\
\hline \hline
\end{tabular}

\section{Beamforming requirements}

The multiple receiving beams of the SCORE principle can be synthesized from a subset of the total FAEs. Each beam, i.e. each receiver output, is constituted by a cluster of $K=5$ out of the 12 FAEs. This means that, as explained later, some elements are shared between the three beams. In general, a Blass matrix has $N$ inputs and $M$ outputs, and every $m$-th output, i.e., each receiving beam can, in principle, employ the input from all the FAEs. Here, a clustered solution is adopted, in which each beam is synthesized employing only a subset of FAEs. The beamforming weights are extracted through an algorithm based on the Minimum Variance Distortionless Response (MVDR) method [22]. Since the amplitude contribution of some weights is negligible, a lower number of elements can be combined contemporarily. This reduction of complexity has a slight but acceptable impact on the antenna gain directivity and smoothness, as shown in Fig. 2, which still present an average beamwidth of $0.93^{\circ}$. To cover the 50 $\mathrm{km}$ of swath width with the required NESZ, all beams have to be reconfigured among 50 possible positions, switching from one to the other each $2.6 \mu$ s. This time takes in account the reconfiguration time, stabilization, dwell time, and acquisition time of the receiver processing unit. As will be discussed in the following, this timing is guaranteed by the selected technology. The reconfiguration of the multiple beams is obtained by applying appropriate beamforming weights on each of the 12 inputs. A Montecarlo analysis performed on the beamforming weights has shown a high sensitivity of the beamforming technique to the weights accuracy. The relative amplitude error of the beamforming weights should remain below $5 \%$ to minimize pointing error and reduce the impact on the signal-to-noise ratio (SNR), which would reduce the radiometric accuracy performance of the SAR system. On the other hand, the beamforming performance is not significantly impacted by random phase errors. Nevertheless the random phase errors shall be constrained to $<10^{\circ}$. All these requirements are listed in TABLE IV, which are fundamental for the realization of the photonic beamformer.

\section{INTEGRATED PHOTONIC BEAMFORMER}

The core activity of the SPACEBEAM project is the design and realization of the beamformer PIC, which will implement the optical beamforming matrix for a SCORE-SAR system. The OBFN is a reconfigurable beamforming matrix that processes the $12 \mathrm{Rx}$ RF signals from the FAEs into up to 3 high-gain beams.

The PIC will be realized using an advanced hybrid technology that combines passive devices with extremely low propagation losses and state-of-the-art InP active devices into 


\begin{tabular}{ll}
\hline \multicolumn{2}{c}{ TABLE V } \\
\multicolumn{1}{c}{ PHOTONIC INTEGRATED CIRCUIT TECHNOLOGY } \\
\multicolumn{1}{c}{ PPECIFICATIONS } \\
\hline \hline \multicolumn{1}{c}{ Varameter } & \multicolumn{1}{c}{ Vybrid TriPleX/InP } \\
\hline Technology & $<0.1 \mathrm{~dB} / \mathrm{cm}$ \\
ADS waveguide propagation loss & $120 \mathrm{~mW}$ \\
Laser output power & $320 \mathrm{~Hz}$ \\
Laser linewidth & $>50 \mathrm{~dB}$ \\
Laser Side Mode Suppression Ratio & $-170 \mathrm{dBc} / \mathrm{Hz}$ \\
Laser Relative Intensity Noise (RIN) & $300 \mathrm{~ns}$ \\
PZT actuator switching time & $25 \mathrm{~V}$ \\
PZT actuator 2 $\pi$ phase shift voltage & $13 \mathrm{~dB}(\mathrm{max} .27 \mathrm{~dB})$ \\
InP SOA gain & $8 \mathrm{~dB}(\mathrm{when}=13 \mathrm{~dB})$ \\
InP SOA noise figure & $3 \mathrm{~V}$ \\
InP MZM half-wave voltage & $6 \mathrm{~dB}$ \\
InP MZM insertion loss & $40 \mathrm{GHz}$ \\
InP MZM EO bandwidth & $0.5 \mathrm{~A} / \mathrm{W}$ \\
InP MUTC PD responsivity & $45 \mathrm{GHz}$ \\
InP MUTC PD bandwidth & \\
\hline \hline
\end{tabular}

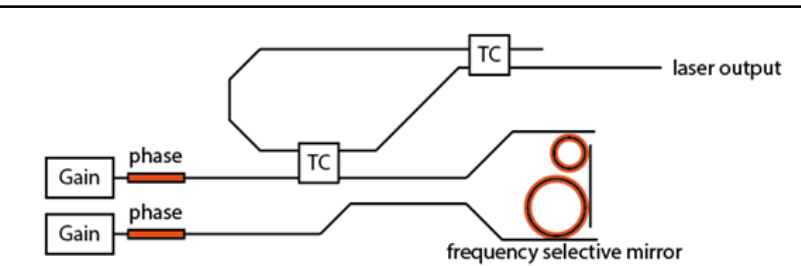

Fig. 3: Schematic concept of the dual gain external cavity laser, realized using a double RSOA InP chip and a cavity with double ring resonator mirror in Vernier configuration realized in TripleX [28].

a single compact assembly. Such technology allows to remove or limit the number of optical interfaces.

In the following, the PIC technology employed for the project demonstrator is introduced. The OBFN is then described and analyzed in details. Finally, the requirements of the PIC packaging are briefly reported.

\section{A. Photonic integrated circuit technology for the beamformer}

The PIC beamformer will be realized using a hybrid integrated photonic technology, merging InP and TriPleX ${ }^{\mathrm{TM}}$. TriPleX is a silicon nitride $\left(\mathrm{Si}_{3} \mathrm{~N}_{4}\right)$ on insulator waveguide technology developed by LioniX. In particular, the key features of one of their silicon nitride planar waveguide technologies, called Asymmetric Double Strip (ADS), are the extremely low loss $(<0.1 \mathrm{~dB} / \mathrm{cm})$, and the bending radius of the integrated optical waveguides $<60 \mu \mathrm{m}$ that have proven to enable high level of planar integration of switches and filters [23].

While gain, modulation and detection cannot be implemented on the TriPleX platform, these can be supplemented by InP chips which can be integrated thanks to the hybridization technology. The InP gain chip can integrate both semiconductor optical amplifiers (SOAs) and phase modulators based on different combination of InGaAsP Multiple Quantum Well (MQW) active regions. Each SOA can achieve up to $27 \mathrm{~dB}$ gain with a saturation output power of typically $100 \mathrm{~mW}$ in the optical C-Band (1530-1570 nm) [24]. The intensity modulators are Travelling Wave Electrode

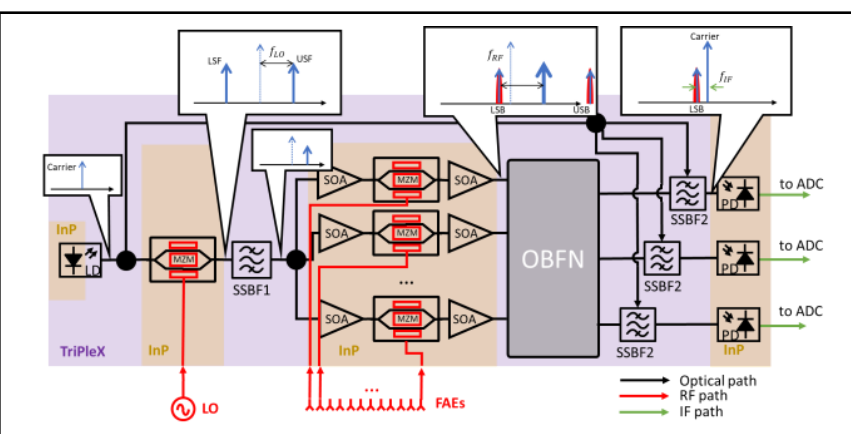

Fig. 4: Architecture of the SPACEBEAM beamformer conceived using a hybrid TriPleX/InP PIC technology. LD: Laser Diode; MZM: MachZehnder Modulator; SSBF(1 and 2). Single-sideband filter; LSF: Lower side frequency; USF: Upper Side Frequency; SOA: Semiconductor Optical Amplifier; OBFN: Optical Beamforming Network; LSB and USB: Signal Lower and Upper Sideband; PD: PhotoDiode; ADC: Analog-to-Digital Converter.

(TWE) Mach-Zehnder Modulator (MZM) also realized in InP, built using MQW optimized for enhancing the QuantumConfined Stark Effect (QCSE) in phase modulators, with a half-wave voltage $\mathrm{V} \pi$ as low as $3 \mathrm{~V}$, with modulation frequencies up to $40 \mathrm{GHz}$ [25], [26]. Finally, the photodetectors (PDs) chip are Modified Uni-Travelling Carrier (MUTC) detectors also realized in InP, with responsivity as high as $0.5 \mathrm{~A} / \mathrm{W}$ and opto-electronic bandwidth above $45 \mathrm{GHz}$ [24], [27]. As can be seen, the available bandwidth of O/E components allows the PIC to be compatible with SAR signals in the full range up to $40 \mathrm{GHz}$.

The InP chips (laser gain section, modulators including amplifiers, and PDs) can be butt-coupled around a larger TriPleX chip including all the passives, so to form a single hybrid PIC assembly that can implement all the functionalities required by the SCORE SAR beamforming receiver. In particular, the laser source is the most notable example of hybrid integration of these two platforms. A InP double reflective SOA (RSOA), on which a High Reflection (HR) coating is applied on one of the chip facet, is buttcoupled to an external cavity including a double ring resonator reflector in Vernier configuration realized in the TripleX platform, as shown in Fig. 3. This laser structure has demonstrated a on-chip power of $120 \mathrm{~mW}(20.8 \mathrm{dBm})$ with a linewidth of $320 \mathrm{~Hz}$ for an emission wavelength of $1550 \mathrm{~nm}$, a Side Mode Suppression Ratio (SMSR) > $50 \mathrm{~dB}$ and a Relativity Intensity Noise (RIN) of $-170 \mathrm{dBc} / \mathrm{Hz}$ [28]. The specifications of the photonic technology and the different components used in the PIC architecture are summarized in TABLE V.

\section{B. Architecture of the photonic beamformer}

Several PIC architectures have been considered for the SPACEBEAM project. One of the most promising is the one shown schematically in Fig. 4. The optical carrier (OC) is generated by the dual gain laser. The OC is then split into two paths. One output of the splitter is used at the final stage as an optical LO to down-convert the RF signals to IF by coupling with each output of the OBFN to three PDs. The second path of the OC goes to an InP Mach-Zehnder modulator (MZM) driven by the electrical $8.3 \mathrm{GHz}$ LO in carrier suppression mode. The modulator output is filtered to select only the upper side frequency (USF) by the first single- 


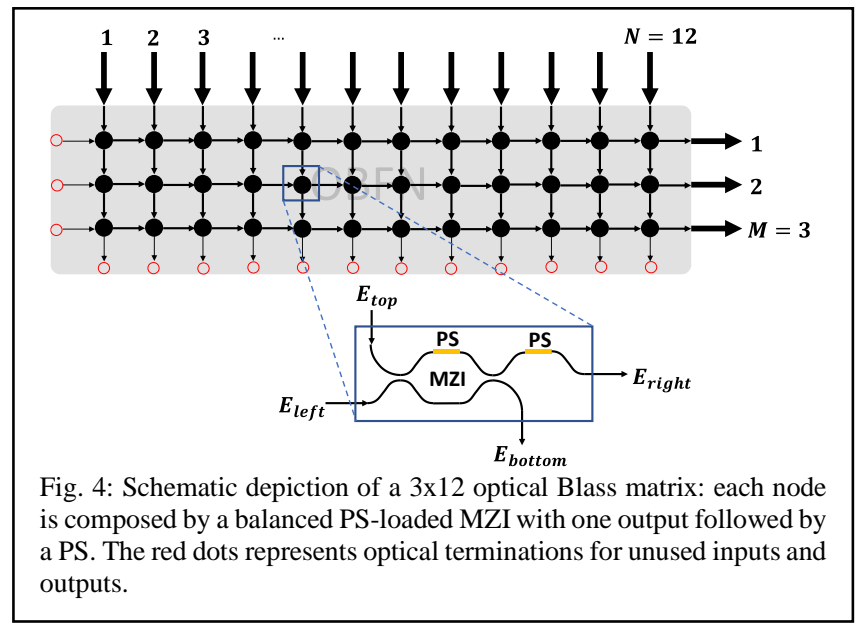

sideband filter (SSBF1). The filter, implemented with an Optical Ring Resonator (ORR) loaded Mach Zehnder Interferometer (MZI), is designed as a periodic $5^{\text {th }}$ order Type II Chebyshev with a passband equal to $36 \%$ of the Free Spectral Range (FSR), being $9.95 \mathrm{GHz}$ for SSBF1 corresponding to a passband of $3.65 \mathrm{GHz}$. The filter center frequency and the FSR have been chosen so that all other optical modulation by-products are rejected. The USF is then split into 12 branches through a cascade of Tunable Couplers (TC)-tree implemented with MZIs. Each split USF line is modulated into another MZM by the received RF signal coming from the corresponding FAE. To compensate the numerous splits of the OC, SOAs (pumped to provide a gain of $13 \mathrm{~dB}$ and a noise figure NF of $8 \mathrm{~dB}$ ) before and after the second MZMs stage boost the USF and the corresponding modulation upper sideband (USB) and lower sideband (LSB), respectively, prior to entering the OBFN. The OBFN, implemented as a Blass matrix (described in detail in the next paragraph), combines the 12 input signals with specific amplitude and phase weights into three outputs. After the OBFN, the LSB is selected by a second single sideband filter SSBF2, designed as SSBF1 with the only difference being the FSR reduced by 3 times to $3.4 \mathrm{GHz}$, resulting in a passband of $0.75 \mathrm{GHz}$. This last filter stage, besides the sideband selection, has two other important functions: it combines the original OC with one output of the OBFN, and it suppresses the out-of-band amplified spontaneous emission (ASE) noise generated by the SOAs. Each filter output is sent to one PD. The down-conversion occurs due to the beating between the OC and the LSB, which will corresponds to an IF signal at $1.3 \mathrm{GHz}$.

\section{The Blass matrix}

The OBFN element included in the PIC beamformer architecture is an electrically tunable optical Blass matrix, whose scheme is shown in Fig. 5. The Blass matrix consists of horizontal and vertical waveguiding lines $(M=3$ rows and $N=12$ columns) that are cross-connected by reconfigurable nodes [16]. Each node is constituted by a MZI loaded with a tunable optical phase shifter (PS), with an additional PS on one of the MZI outputs. The PS-loaded MZI acts as a tunable coupler, enabling the application of an arbitrary amplitude

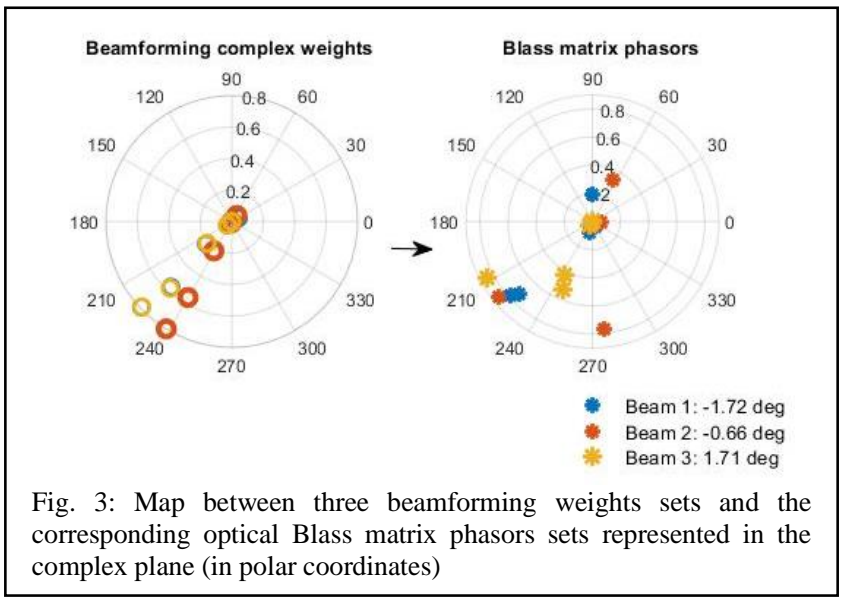

scaling of the signal, whereas the output PS enables the control in phase. The ideal (lossless) transfer matrix of each node is

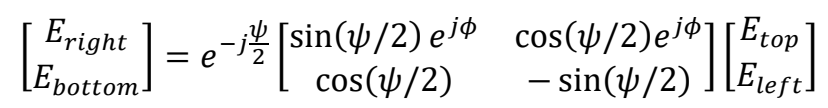

where $E_{\text {right }}, E_{\text {bottom }}, E_{\text {top }}, E_{\text {left }}$ are the electric fields labeled following the convention of Fig. 5 while $\psi$ and $\phi$ are the phase-shifts of the tunable coupler PS and the output PS respectively. By providing two control parameters for each node, this network configuration enables the implementation of any passive linear $M \times N$ transformation $S$ of the inputs.

In the SPACEBEAM application, the Blass matrix must accept 12 input signals, apply an appropriate beamforming weight to each carrier and combine them into 3 outputs, resulting in a matrix with 36 nodes. Following the convention of Fig. 4, this means that each of the 3 rows of the matrix applies a set of 12 beamforming complex weights ((i.e., with amplitude and phase) that corresponds to a specific beam direction. Since each node is controlled by two PSs, the Blass matrix requires the synthesis of 72 phase controllers in total. By using the network synthesis algorithm described in [16], it is possible to map the beamforming weights matrix $S=$ $\left[s_{m n}\right]$ with two matrices containing all the values of $\psi_{m n}$ and $\phi_{m n}$, that can be represented by the phasor matrix as

$$
B=\left[\left|r_{m n}\right| e^{j \phi_{m n}}\right], \quad 1 \leq m \leq 3 ; 1 \leq n \leq 12
$$

with $\left|r_{n m}\right|^{2}=\left|\sin \left(\psi_{n m} / 2\right)\right|^{2}$ being the fraction of power from the top $n(m-1)$ input to the right $m n$ output through the MZI tunable coupler. As an example, Fig. 6 shows the relationship between one of the 50 possible $S$ matrices configurations and the corresponding $B$ matrix for a specific set of beam directions required by the current application.

If the number of employed matrix elements would be an integer number $K \leq N / M$, then the $M$ output beams would be completely independent from each other. Given the presented structure, the maximum value that $K$ can reach to meet this condition is $K_{\max }=4$. Since, in our case, the $M=3$ output beams are the result of the beamforming employing the signals from $K=5 \geq K_{\max }$ FAEs, and the 3 beams are simultaneously generated, some elements of one row of the matrix interact with elements of the adjacent rows. As shown in Fig. 5, each $(m, n)$-th matrix node has two inputs, one from the (m,n-1)-th node (i.e., the previous node on the same row), the other from the $(m-1, n)$-th node (i.e., the corresponding 


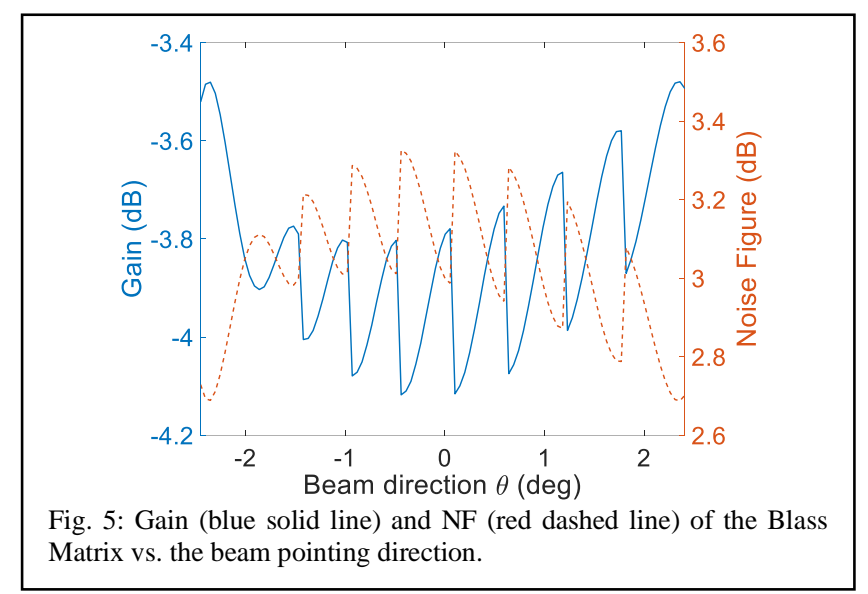

node on the upper row) and two outputs, one to the $(m, n+1)$ th node (i.e., the following node on the same row) and one to the $(m+1, n)$-th node (i.e., to the corresponding node on the lower row). Since groups of 5 nodes are activated for beamforming on each row, $K-K_{\max }=1$ active element in the middle row receives an input from the upper row and 1 other element sends an output to the lower row; this means that the operation of this element is affected by the operation of an element from another group and, at the same time, it impacts on the operation of another element of another group. This way, the beamforming operations on the three beams are not independent.

The contribution of the Blass matrix placed in the RF link chain is not trivial due to its multiple-input/multiple-output (MIMO) nature. Indeed, even if the Blass matrix is a passive matrix with no active components, the determination of its gain and NF must consider the combination of different signals on the same output, taking into account that signals are summed coherently, whereas the noise sources sum up incoherently. Let us define $E_{\text {in }}$ as the total RF modulated optical field from a cluster of $K=5$ inputs (coming from the MZMs), while $E_{\text {out }, m}$ defined as one of the $M=3$ optical outputs toward the PD. Then, let us consider a Blass matrix that realizes the beamforming complex weight matrix $S(\theta)$ for a specific beam direction $\theta$. Under these definitions, the gain of the $\mathrm{m}^{\text {th }}$ Blass matrix output can be computed:

$$
\begin{array}{r}
G_{B M, m}(\theta)=\frac{\left|E_{\text {out }, m}\right|^{2}}{\left|E_{\text {in }}\right|^{2}}=\frac{\left|\frac{E_{\text {in }}}{\sqrt{K}} \sum_{k=1}^{K} s_{k m}(\theta)\right|^{2}}{\left|E_{\text {in }}\right|^{2}} \\
=\frac{1}{K}\left|\sum_{k=1}^{K} s_{k m}(\theta)\right|^{2},
\end{array}
$$

with the $s_{k m}(\theta)$ complex weights associated to each matrix node. In this application, on each matrix row, only 5 out of $12 s_{k m}(\theta)$ have non-zero amplitude. Therefore, it is important to notice that the gain is computed considering an input power equal to $5 / 12$ of the total power at the antenna. On the other hand, the NF of the $\mathrm{m}^{\text {th }}$ output is expressed as:

$$
N F_{B M, m}(\theta)=\frac{K \sum_{k=1}^{K}\left|s_{k m}(\theta)\right|^{2}}{\left|\sum_{k=1}^{K} s_{k m}(\theta)\right|^{2}}=\frac{1}{G_{B M, m}} \sum_{k=1}^{K}\left|s_{k m}(\theta)\right|^{2} .
$$

The single output gain $G_{B M}$ and noise figure $N F_{B M}$ of the Blass matrix have been computed for all the expected 50

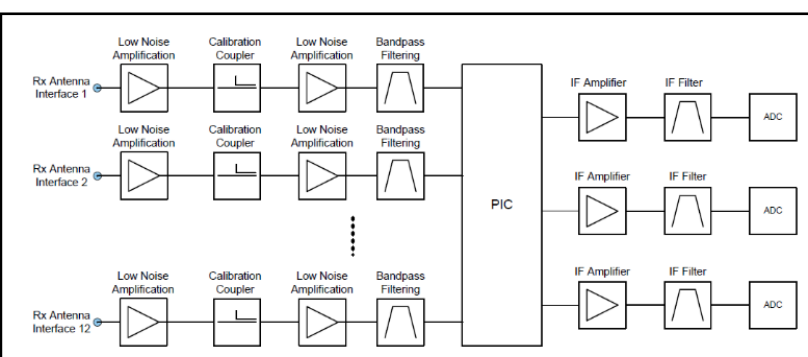

Fig. 7: Block diagram of the receiver RF front-end from the antenna FAEs to the ADCs

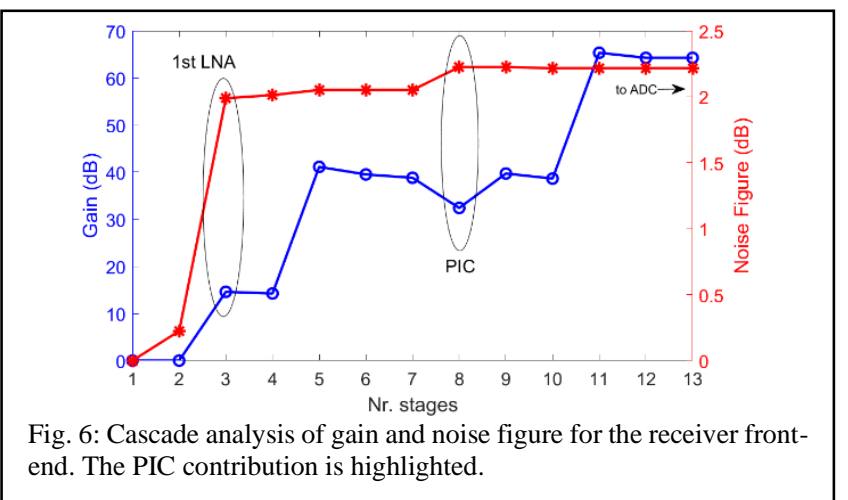

pointing directions of the beams required by the application, which are between $-2.5^{\circ}$ and $2.5^{\circ}$ and the related plots are reported in Fig. 7, resulting in an average gain of $-3 \mathrm{~dB}$, and a NF of $3 \mathrm{~dB}$.

Each pointing direction $\theta$ requires a different set of weights, which are obtained by controlling the PSs in the matrix. To control the 72 PSs, a recently proposed technique will be used, exploiting Lead Zirconate Titanate (PZT) piezoelectric actuators deposited on top of the TriPleX waveguides [29]. This solution is much faster than the standard thermal controls, allowing for a $2 \pi$ phase shift in about 300 ns (instead of hundreds of $\mu \mathrm{s}$ ), thus permitting the fast beamforming required by the SPACEBEAM application. Each actuator is 15 -mm long, with a $2 \pi$-voltage of $25 \mathrm{~V}$. Moreover, the power consumption of the PZT is in the order of hundreds of $\mathrm{nW}$ [29], whereas a single thermal control may require up to hundreds of $\mathrm{mW}$. Considering that the total number of controls needed by the presented PIC is 205, the estimated max power consumption due to PZT controls is merely 121 $\underline{\mu W}$. Therefore, PZT is a great candidate for space applications where power consumption and heat dissipation are critical issues.

Finally, the required accuracy of the phase-shifts has also been assessed. According to the system specifications, the amplitude error of the beamforming weights in the worst case should be below $5 \%$, whereas phase errors shall be below $10^{\circ}$. To estimate the accepted statistical distributions of the $B$ matrix containing the values of the phase-shifts, a Montecarlo analysis has been carried out by iterating the network synthesis algorithm on a $S$ matrix with a random error applied as

$$
S_{\text {err }}=\left(I+G_{5 \%}\right) e^{j U_{10^{\circ}} S}
$$

with $I$ being the identity matrix, $G_{5 \%}$ being an amplitude error with zero-mean normal distribution and 5\% standard deviation, and $U_{10^{\circ}}$ being a phase error uniform distribution, 
with zero mean and $10^{\circ}$ standard deviation. The derived smallest standard deviation among all phase-shift values is of 0.02 rad. The analysis, carried out on different sets of beamforming weights, provides an upper limit to the phaseshifters accuracy. Considering that the PZT actuators that will drive the Blass matrix phase shifters have a $2 \pi$ voltage of around $30 \mathrm{~V}$, the maximum error $\epsilon$ in the voltage driving the PZT actuators that will ensure an amplitude error $<5 \%$ in the beamforming weights is $\epsilon=(0.02 / 2 \pi) 30 \mathrm{~V}=95.5 \mathrm{mV}$. This value provides the minimum precision for the control electronics that will drive the PIC.

\section{Architecture performance estimation}

During the design phase of the SPACEBEAM beamformer, several architectures have been considered (similar to that shown in Fig. 3) by changing the position and the number of the SOAs, and the way the optical LO is generated. The different possible architectures have been evaluated through simulations in VPI Transmission Maker ${ }^{\mathrm{TM}}$. The simulation shows also the evolution of both the optical and electrical signals that propagate through the architecture building blocks making it possible to evaluate the contribution of each element in terms of SNR, frequency response and power consumption. In particular, gain and NF are of primary importance for the design of the entire RF receiver system, both front-end and back-end. The gain has been calculated as the difference between the IF signal power at $1.3 \mathrm{GHz}$ from the PD output and the total input RF power to 5 of the 12 MZMs. The NF, on the other hand, is the difference between the SNR at the PIC output and 5 RF inputs limited by thermal noise at room temperature (i.e. $290 \mathrm{~K}$ ) integrated over the signal bandwidth of $390 \mathrm{MHz}$. The simulations have been setup so that a number of assumptions could be made: a) SOAs are assumed to be working in the linear regime (valid for optical inputs below $4 \mathrm{dBm}$ ) with 13 $\mathrm{dB}$ gain and $8 \mathrm{~dB}$ NF over all frequencies; b) The MZMs phase modulators have a linear response to the applied signal ( $\Delta \phi=\pi V_{R F} / V_{\pi}$ for any value with $V_{\pi}=3 \mathrm{~V}$ with no dependence on frequency) and do not present saturation effects (valid for optical inputs below $17 \mathrm{dBm}$ ); c) PDs have a flat responsivity $(0.5 \mathrm{~A} / \mathrm{W})$ over all frequencies (dark current and shot noise are enabled); d) the MZI tunable couplers of the Blass matrix have infinite extinction ratios (only susceptible to fabrication tolerances); e) the optical SSBFs are ideal periodic $5^{\text {rd }}$-order Chebychev filters; f) the laser does not present any side-modes in the frequency range of interest.

Under these assumptions, among all the considered architecture variants, the scheme reported in Fig. 3 exhibited the best performance, resulting in an estimated gain of $-12 \mathrm{~dB}$ and a NF of $30 \mathrm{~dB}$. These values fit with the targeted overall system performance in terms of expected dynamic range. It was observed that the NF is significantly reduced by the SSBF2 before the PD, which rejects most of the ASE produced by the second stage of SOAs. According to simulations, the ASE-ASE beat noise turns out to be by far the dominant source of noise at IF, with an evident dependence on the optical filter bandwidth. Conversely, the ASE-signal beat noise is not as strong due to the very low
TABLE VI

PHOTONIC INTEGRATED CIRCUIT BEAMFORMER SPECIFICATIONS

\begin{tabular}{ll}
\hline \hline \multicolumn{1}{c}{ Parameter } & \multicolumn{1}{c}{ Value / Range } \\
\hline Input RF frequency & 5 to $40 \mathrm{GHz}$ \\
Output IF frequency & 1 to $5 \mathrm{GHz}$ \\
Integration bandwidth & $390 \mathrm{MHz}$ \\
Gain (from 5 input to 1 output) & $-12 \mathrm{~dB}$ \\
Noise Figure (from 5 input to 1 output) & $30 \mathrm{~dB}$ \\
Spurious Free Dynamic Range & $55 \mathrm{~dB}$ \\
Maximum power consumption & $3.5 \mathrm{~W}$ \\
\hline \hline
\end{tabular}

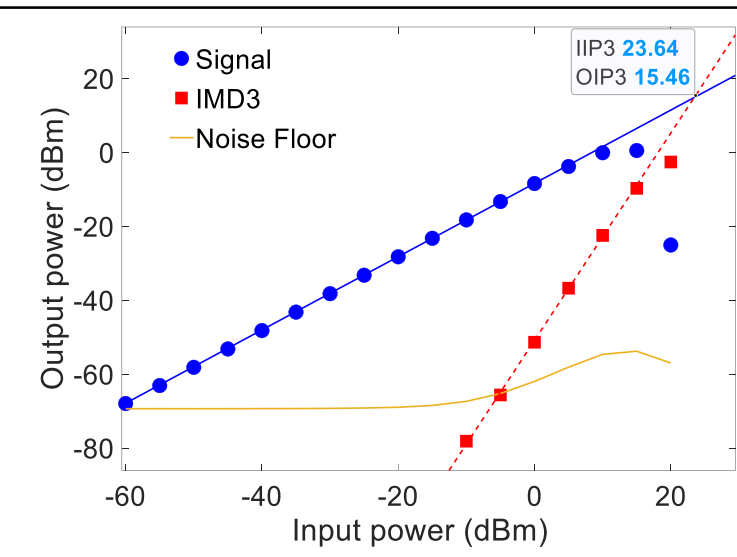

Fig. 8: Two-tone test for the analysis of the spurious-free dynamic range of the PIC beamformer.

signal level, and its effect becomes appreciable only due to the presence of the SSBFs.

Compared to other microwave photonics architectures [10], a $-12 \mathrm{~dB}$ gain is high, considering the number of functions performed by the presented hybrid PIC. Even if the NF value might sound large, although reasonable for a photonics-based down-conversion, the PIC is placed after other RF devices with high gain and lower NF, and this does not affect much the NF of the whole SAR receiver, whose simplified front-end block diagram is shown in Fig. 8. Each FAE is equipped with a dedicated amplification chain, which is responsible for the amplification of the weak signals coming from the antenna. The IF chain amplifies the beamformed signals up to the power level required by the ADCs. Since the gain and NF of the PIC OBFN have been assessed, it is possible to determine its contribution in the RF receiver link of the SCORE-SAR system. As it can be observed from the cascade analysis of Fig. 9, despite the large NF value of the PIC, its contribution to the overall NF of the RF front-end is negligible with a value of $2.2 \mathrm{~dB}$.

Besides gain and NF, the spurious-free dynamic range (SFDR) was also estimated by running a two-tone (spaced of $100 \mathrm{MHz}$ ) test analysis. The results from the simulation are shown in Fig. 10 where, considering the signal (blue circles) and the $3^{\text {rd }}$-order intermodulation product (IMD3, red squares), an output intercept point of the $3^{\text {rd }}$ order (OIP3) as high as $15.5 \mathrm{dBm}$ is obtained. The value of the SFDR turns out to be $55 \mathrm{~dB}$ for an integration bandwidth of $390 \mathrm{MHz}$, which is well enough for the dynamic range required by the SCORE SAR application. It is worth noticing that the noise floor (yellow line) is not flat but exhibits a maximum around $15 \mathrm{dBm}$ input power. This is due to the ASE-signal beating 
summing with the ASE-IMD3 beating. The noise floor starts increasing when the latter becomes stronger, and it weakens when the two frequency components start saturating. However, it does not roll off as fast as the signal, because the IMD3 power decreases much more slowly.

As a final note, the architecture design started considering different possible schemes. One of the most discriminant factor between different architecture variants was the number of SOAs deployed: indeed, they strongly impact the overall power consumption of the entire PIC beamformer assembly. Strict power consumptions requirements lead to different grades for the SWaP and the need to develop more advanced packaging solutions than the one currently adopted and described in the following subsection. The architecture considered in Fig. 4 has 24 SOAs; other considered schemes employed 12 SOAs only, but at the cost of a lower gain, and even higher NF, which made their performance less suitable to our system requirements. Moreover, the lower number of SOAs does not significantly impact on the overall SAR system power consumption. In fact, as far as the optical signals are weak, as in this case, the absorption of the SOAs is low, and they consume much lower power than the RF front-end of the SAR, which is expected to contribute for several $\mathrm{kW}$. A preliminary analysis of the total power consumption of the hybrid PIC assembly has been carried out through a cascade analysis of the architecture. The assumption made for the analysis is that each SOA requires an electrical power 10 times the output optical power. Under this assumption, the laser absorbs $1 \mathrm{~W}$, whereas the first stage of 12 SOA consumes $2.2 \mathrm{~W}$. The second stage of SOAs, which amplify very weak signals, consumes in total $62 \mathrm{~mW}$. The maximum power consumption of the MZM is $24 \mathrm{~mW}$ each, totaling $288 \mathrm{~mW}$ for 12 MZMs. The power consumption of the TriPleX chip, which hosts all the filters, the switches and the beamforming matrix, is negligible. As already mentioned, each PZT actuator consumes $300 \mathrm{nW}$ per $\pi$ shift. Having 205 PZT controls on the TripleX chip, a maximum of $123 \mu \mathrm{W}$ is expected. We can thus claim that the total power consumption of the hybrid assembly will be approx. $3.5 \mathrm{~W}$. The estimated performance of the PIC architecture are reported in TABLE VI.

\section{E. PIC Packaging}

A crucial aspect of the proposed system lies in the packaging, which should not only provide a seamless electrical DC and RF interface for the hybrid assembly, but also ensure the required ruggedness for the specific targeted application. As any equipment intended to be mounted on a satellite, the SCORE-SAR system of this project must be capable of absorbing extreme mechanical and thermal shocks, as well as being shielded from radiations. Therefore, a careful definition of a hermetic packaging specifications has been necessary. The packaging will be developed and carried out ensuring full compatibility with the state-of-the-art packaging procedures of the European Packaging Pilot Line PIXAPP [30] and will undergo environmental testing for demonstrating the space compliance of the SPACEBEAM solution.

The hybrid PIC-assembly consists of four different sections:

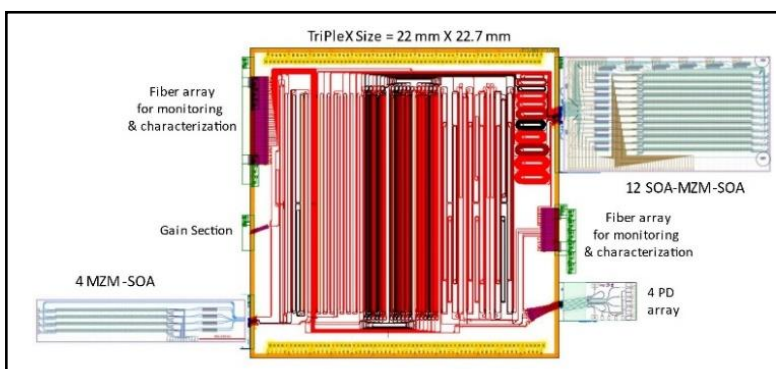

Fig. 9: Hybrid TriPleX/InP PIC assembly layout for the SPACEBEAM beamformer. The block on the left side, at the bottom of the picture, containing the first MZM-SOA, comes from the foundry as a chip including 4 MZM-SOA, although only one of them is employed.

- Main TriPleX chip including all passive functions, with a size of $22 \mathrm{~mm} \times 27 \mathrm{~mm}$, interfaced with the three InP chips listed below,

- InP chip including $1 \mathrm{MZM-SOA}$ array with a size of $15 \mathrm{~mm}$ x $3 \mathrm{~mm}$,

- InP chip hosting the 12 SOA-MZM-SOA with a size of 16 $\mathrm{mm} \times 8 \mathrm{~mm}$,

- InP chip hosting the PDs, with a size of $3 \mathrm{~mm} \times 3 \mathrm{~mm}$.

The total assembly size, whose layout is shown in Fig. 11, is $53 \mathrm{~mm} \times 30 \mathrm{~mm}$.

Compatibility with high-speed electronics will be ensured by low-temperature co-fired ceramic (LTCC) boards, a packaging solution for RF modules particularly attractive for space applications: LTCC has been identified as the best solution in terms of mechanical stability, with a flexural strength between $200 \mathrm{MPa}$ and $400 \mathrm{MPa}$, and a Young's modulus of elasticity in the range $75-175 \mathrm{GPa}$, combined with a thermal conductivity $\sim 3 \mathrm{~W} / \mathrm{mK}$. The LTCC assembly will be performed resorting to specific adhesives, since adhesives are much more flexible than solder and, therefore, better able to withstand vibrations.

As for the external interfaces, only DC and RF electrical interfaces will be present with no optical inputs or outputs. The PIC package design will host a total of $16 \mathrm{RF}$ feedthrough ports with $50 \mathrm{Ohm}$ characteristic impedance. One is for the electrical LO at $8.3 \mathrm{GHz}$ for the first MZM stage for the generation of the USF carrier. Provided that the MZM will be driven in carrier suppression mode, driving it with a voltage larger than $2 \mathrm{~V} \pi$ (i.e. with electrical power $>23$ $\mathrm{dBm}$ for a $50 \mathrm{Ohm}$ load) does not carry any advantage, and will only consume more power. $12 \mathrm{RF}$ ports are the inputs for the X-band signals from the antenna FAEs to the second MZM stage. The expected power impinging on each antenna FAE is in the range $-89.6 \mathrm{dBm}--57.1 \mathrm{dBm}$, brought up in the range $-53.7 \mathrm{dBm}--21.4 \mathrm{dBm}$ by the receiver front-end amplifier chain. Finally, 3 IF feedthrough ports are required for the 3 PDs outputs, at $1.3 \mathrm{GHz}$. The output power range from the IF feedthrough ports is $-66 \mathrm{dBm}--34 \mathrm{dBm}$. The signal output will be amplified by a $32 \mathrm{~dB}$-gain IF amplifier, to shift the power in the $-36.4 \mathrm{dBm} \div-4.1 \mathrm{dBm}$ range at the ADC input. All these 16 ports will have ground-signalground (GSG) coplanar interfaces for connections to the LTCC. Due to the large channel numbers, extra care must be taken to ensure that the PIC and the LTCC can be physically 
TABLE VII

PIC PACKAGING REQUIREMENTS

\begin{tabular}{ll}
\hline \multicolumn{1}{c}{ Parameter } & \multicolumn{1}{c}{ Value / Range } \\
\hline Technology & Gold box with LTCC \\
PIC Assembly area & $53 \mathrm{~mm}$ x $30 \mathrm{~mm}$ \\
Input RF frequency & $9.6 \mathrm{GHz}$ \\
Input LO frequency & $8.3 \mathrm{GHz}$ \\
Output IF frequency & $1.3 \mathrm{GHz}$ \\
Signal bandwidth & $390 \mathrm{MHz}$ \\
RF transmission line type & Coplanar waveguide $(\mathrm{CPW})$ \\
Input RF power range & $-36.4 \mathrm{dBm}$ to $-4.1 \mathrm{dBm}$ \\
Output IF power range & $-66 \mathrm{dBm}$ to $-34 \mathrm{dBm}$ \\
Nr. Optical I/O & 0 \\
Nr. RF inputs & $13(12 \mathrm{FAEs}+1 \mathrm{LO})$ \\
Nr. RF outputs & 3 \\
Nr. DC controls & $205(72$ for the Blass matrix) \\
Package leak rate & $<1 \times 10^{-8}$ atm $\mathrm{cm}^{3} / \mathrm{s}$ \\
Radiation hardness & $>35 \mathrm{krad}$ \\
Shock tolerance & $<1500 \mathrm{~g}$ (for $0.5 \mathrm{~ms})$ \\
Vibration tolerance & $<20 \mathrm{~g}$ (in the 22.5 to $40 \mathrm{~Hz})$ \\
\hline
\end{tabular}

connected while maintaining impedance matching, at the same time minimizing parasitic losses.

The PIC will also need a large number of DC controls. They will be necessary to drive the PZT actuators for the beamformer phase shifters, to control the resonator structures present in the laser and the filters, to apply the bias to the MZMs and to the PDs, and to inject the pump current for the SOAs. The required number of DC electrical feedthroughs is around 150, half of which (72 pads) will be needed for the PZT actuators driving the Blass matrix implementing the beamforming functionality.

The assembly will be enclosed in a hermetically sealable gold box together with the LTCC boards as interposers that will fill the space gap between the PIC and the gold box edges. A thermo-electric cooler (TEC), to guarantee a stable working temperature, will be positioned inside the box, underneath the PIC. The package will be hermetic with a leak rate $<1 \times 10-8 \mathrm{~atm} \mathrm{~cm} / \mathrm{s}$, and a radiation hardness $>35 \mathrm{krad}$, a typical value considering the targeted LEO orbit. The total dimension of the packaged PIC assembly, including the goldbox hosting the PIC assembly, and the board for the DC and RF interfaces is expected to be $15 \times 10 \mathrm{~cm}^{2}$. Finally, the whole package will undergo environmental trials to ensure its compatibility with space-grade requirements, including also shocks with $1500 \mathrm{~g}$ magnitude and $0.5 \mathrm{~ms}$ duration, as well as vibrations with frequencies in the range $22.5 \mathrm{~Hz}-40 \mathrm{~Hz}$, and an intensity reaching $20 \mathrm{~g}$. The requirements for the PIC packaging are summarized in TABLE VII.

\section{CONCLUSION}

This paper has presented the design and performance estimation of an integrated-photonics RF beamformer, aimed at enabling a multi-beam SCORE-SAR for satellite Earth observation. The activity is the core of the SPACEBEAM project and considers the hybrid integration of InP chips including the active components, with a TriPleX chip including the passive components and the Blass matrix implementing the beamforming network. The system simulation estimates a PIC gain of $-12 \mathrm{~dB}$ and a NF of $30 \mathrm{~dB}$, guaranteeing a dynamic range of $55 \mathrm{~dB}$, well above the specific requirement of the application. The control of the
Blass matrix implemented through the recently developed PZT piezo-electric actuators allows for a very fast beamforming, permitting the SAR to distinguish between 50 different view angles along a very wide swath of $50 \mathrm{~km}$. In addition, strict packaging requirements have been defined to allow the hybrid PIC to reach the space environment compliance.

In the SAR application, the photonics-assisted beamforming can ensure a frequency-agnostic operation with unchanged performance over the whole interval of all useful $\underline{\mathrm{RF}}$ frequencies for $\mathrm{EO}$ (up to $40 \mathrm{GHz}$ ), with high precision and continuous beam steering. For higher frequency applications, the proposed architecture requires a consistent scaling, although this is primarily due to mission or system requirements that are not strictly related to the adopted implementation. On the other hand, the proposed approach is suitable to be extended to higher frequency, maintaining all the offered advantages in terms of SWaP. The photonic beamformer, besides controlling the direction of the received beam, also implements a photonics-based down-conversion of the received RF signals, so that the beamformed signal at its output can be directly acquired by an ADC, without any further RF down-conversion stage. The expected very compact form factor of the hybrid PIC-assembly of $15 \mathrm{~cm}^{2}$, the low power consumption of $3.5 \mathrm{~W}$, and a spurious-free dynamic range (SFDR) of $55 \mathrm{~dB}$, make this microwave photonic receiver suitable for the targeted SCORE-SAR mission requirements.

The outcome of this activity is expected to open up several new possibilities in other Earth observation applications, showing the potential to become a new standard component for the next spaceborne SARs.

\section{REFERENCES}

[1] M. Chini, R. Pelich, R. Hostache P. Matgen, C. Lopez-Martinez, "Towards a $20 \mathrm{~m}$ Global Building Map from Sentinel-1 SAR Data" MDPI Remote Sens. 10 (11), p. 1833 (2018).

[2] H.-D. Guo, L. Zhang, L.-W. Zhu, "Earth observation big data for climate change research", in Advances in Climate Change Research 6 (2), pp. 108-117 (2015).

[3] A. Moreira et al., "A tutorial on synthetic aperture radar," IEEE Geosci. Remote Sens. Mag., vol. 1, no. 1, pp. 6-43, Mar. 2013, doi: 10.1109/MGRS.2013.2248301.

[4] P. A. Rosen e R. Kumar, «NASA-ISRO SAR (NISAR) Mission Status», in 2021 IEEE Radar Conference (RadarConf21), mag. 2021, pagg. 1-6. doi: 10.1109/RadarConf2147009.2021.9455211.

[5] A. Sezer, O. Çelikkaya B. Hassoy, "Microsatellite constellation for earth observation missions," 8th International Conference on Recent Advances in Space Technologies (RAST), Istanbul, Turkey, pp. 279284 (2017).

[6] A. Moreira, M. Zink, M. Bartusch, A. E. Nuncio Quiroz, e S. Stettner, «German Spaceborne SAR Missions», in 2021 IEEE Radar Conference (RadarConf21), mag. 2021, pagg. 1-6. doi: 10.1109/RadarConf2147009.2021.9455326.

[7] M. V. Drummond et al., "Dimensioning of a multibeam coherent photonic beamformer fed by a phased array antenna," Opt. Express, $O E$, vol. 26, no. 5, pp. 6158-6171, Mar. 2018, doi: 10.1364/OE.26.006158.

[8] P. Ghelfi et al., "A fully photonics-based coherent radar system," Nature, vol. 507, no. 7492, Art. no. 7492, Mar. 2014, doi: 10.1038/nature13078.

[9] G. Serafino et al., "Toward a New Generation of Radar Systems Based on Microwave Photonic Technologies," J. Lightwave Technol., JLT, vol. 37, no. 2, pp. 643-650, Jan. 2019. 
[10] D. Marpaung, J. Yao, J. Capmany, "Integrated microwave photonics", Nature Photon. 13, pp. 80-90 (2019).

[11] C. Roeloffzen et al., "Integrated microwave photonics for 5G," 2018 Conference on Lasers and Electro-Optics (CLEO), San Jose, CA, USA, 2018, pp. 1-2.

[12] G. Serafino et al., "High-Performance Beamforming Network Based on Si-Photonics Phase Shifters for Wideband Communications and Radar Applications," in IEEE Journal of Selected Topics in Quantum Electronics, vol. 26, no. 5, pp. 1-11, Sept.-Oct. 2020.

[13] M. Krainak et al., "Integrated photonics for NASA applications," in Components and Packaging for Laser Systems V, Mar. 2019, vol. 10899, p. 108990F, doi: 10.1117/12.2509808.

[14] "SPACEBEAM - Space SAR system with reconfigurable integrated photonic beamforming" https://www.spacebeam-project.eu/ (accessed Apr. 11, 2021)

[15] M. Reza et al., "Design of an Integrated-Photonics RF Beamformer for Multi-Beam Satellite Synthetic Aperture Radar," International Topical Meeting on Microwave Photonics (MWP), Matsue, Japan, 2020, pp. 87-90.

[16] C. Tsokos et al., "Analysis of a Multibeam Optical Beamforming Network Based on Blass Matrix Architecture," Journal of Lightwave Technology, vol. 36, no. 16, pp. 3354-3372, Aug. 2018, doi: 10.1109/JLT.2018.2841861.

[17] Soumekh, Mehrdad. Synthetic aperture radar signal processing. Vol. 7. New York: Wiley, 1999.

[18] M. Younis, C. Fischer, W. Wiesbeck, "Digital beamforming in SAR systems," IEEE Transactions on Geoscience and Remote Sensing, vol. 41, no. 7, pp. 1735-1739, Jul. 2003, doi: 10.1109/TGRS.2003.815662.

[19] N. Gebert, G. Krieger, A. Moreira, "Digital Beamforming for HRWSSAR Imaging: System Design, Performance and Optimization Strategies," in 2006 IEEE International Symposium on Geoscience and Remote Sensing, Jul. 2006, pp. 1836-1839, doi: 10.1109/IGARSS.2006.474.

[20] M. Younis, S. Huber, A. Patyuchenko, F. Bordoni, G. Krieger, "Performance Comparison of Reflector- and Planar-Antenna Based Digital Beam-Forming SAR,” International Journal of Antennas and
Propagation, vol. 2009, p. e614931, Jun. 2009, doi: $10.1155 / 2009 / 614931$.

[21] Ulaby, Fawwaz, M. Craig Dobson, José Luis Álvarez-Pérez. Handbook of radar scattering statistics for terrain. Artech House, 2019.

[22] H.L.V. Trees, "Optimum Array Processing”, John Wiley \& Sons, Inc., 2002.

[23] C. G. H. Roeloffzen et al., "Low-Loss Si3N4 TriPleX Optical Waveguides: Technology and Applications Overview," IEEE Journal of Selected Topics in Quantum Electronics, vol. 24, no. 4, pp. 1-21, Jul. 2018, doi: 10.1109/JSTQE.2018.2793945.

[24] K. V. Gasse, R. Wang, e G. Roelkens, «27 dB gain III-V-on-silicon semiconductor optical amplifier with $>17 \mathrm{dBm}$ output power», Opt. Express, $O E$, vol. 27, n. 1, pagg. 293-302, gen. 2019, doi: 10.1364/OE.27.000293.

[25] F. M. Soares et al., "InP-Based Foundry PICs for Optical Interconnects," Applied Sciences, vol. 9, no. 8, Art. no. 8, Jan. 2019, doi: 10.3390/app9081588.

[26] K.-O. Velthaus et al., «High performance InP-based Mach-Zehnder modulators for 10 to $100 \mathrm{~Gb} / \mathrm{s}$ optical fiber transmission systems», in IPRM 2011 - 23rd International Conference on Indium Phosphide and Related Materials, mag. 2011, pagg. 1-4.

[27] P. Runge et al., «Linearity of Waveguide Integrated Modified UniTravelling Carrier Photodiode Arrays», IEEE Photon. Technol. Lett., vol. 31, n. 3, pagg. 246-249, feb. 2019, doi: 10.1109/LPT.2018.2890015.

[28] K.-J. Boller et al., «Hybrid Integrated Semiconductor Lasers with Silicon Nitride Feedback Circuits», Photonics, vol. 7, n. 1, Art. n. 1, mar. 2020, doi: 10.3390/photonics7010004.

[29] N. Hosseini et al., "Stress-optic modulator in TriPleX platform using a piezoelectric lead zirconate titanate (PZT) thin film," Opt. Express, OE, vol. 23, no. 11, pp. 14018-14026, Jun. 2015, doi: 10.1364/OE.23.014018

[30] Advanced Photonics Packaging and System Integration Services within EUROPRACTICE, ver. 1.5 (Tyndall National Institute). 